\title{
A PRELIMINARY CHECK-LIST OF THE HEPATICS OF KAMCHATKA PENINSULA (RUSSIAN FAR EAST)
}

\section{ПРЕДВАРИТЕЛЬНЫЙ СПИСОК ПЕЧЕНОЧНИКОВ ПОЛУОСТРОВА КАМЧАТКА (РОССИЙСКИЙ ДАЛЬНИЙ ВОСТОК)}

\author{
V. A. BAKALIN ${ }^{1}$ \\ В. А. БАКАЛИН ${ }^{1}$
}

Abstract

\begin{abstract}
A check-list of the liverworts of Kamchatka Peninsula (Russian Far East) has been compiled for the first time. It contains 158 species, including 78 new records for the peninsula. One species is recorded for the first time in Russia (Nardia unispiralis Amakawa), two species are new for Asia, and 4 are new for East Asia. There are also some rare Asian species. Lophozia latifolia R. M. Schust. is synonymized with L. propagulifera (Gottsche) Steph.
\end{abstract}

Резюме

Впервые составлен список печеночников Камчатки. Он включает 158 видов, 78 из которых приводится для флоры полуострова впервые. Среди найденных один вид впервые выявлен во флоре России (Nardia unispiralis Amakawa); раннее он считался Японским эндемом. Еще два вида являются новыми для Азии и еще четыре - новыми для Восточной Азии. Показано, что Lophozia latifolia R. M. Schust. является синонимом $L$. propagulifera (Gottsche) Steph.

HISTORICAL REVIEW

The study of the liverworts of the Kamchatka Peninsula started with G. Wahlenberg's (1811) paper, where he recorded 8 species for the peninsula, but without citing any collection localities. Apparently these collection were made by $\mathrm{H}$. Tilesius near Avacha Bay (localities 15 or 16 in Fig. 1.). The best finding was Asterella saccata, new to science. In $1927 \mathrm{H}$. W. Arnell published an account of the hepatics collected by E. Hulten during the expedition on thesteamship "Commander Bering" (1920-1922). Arnell enumerated 28 species, including Nardia kamtschatica Arnell new to science (in 1976 it was synonymised with $N$. assamica by J. Váňa). In 1970 H. Persson published results of a study of the collection of Kamchatian bryophytes made by Hulten and R. Malaise (these specimens were collected in 1920-1926 and by 1970 were still unidentified). Up to 1970 about 50 species were known from Kamchatka. Persson (1. c.: 209) estimated that the number of "known bryophytes from Kamchatka will increase by some 30-40\%". However, current research has shown that this is an underestimate, and the hepatic flora of Kamchatka is considerably richer.
The next publication on Kamchatian hepatics was in 2001 (Blagodatskich \& Duda, 2001) with a list of the species collected by L. S. Blagodatskich in 1985. Blagodatskich had collected about 50 specimens in Kronotsky State Reserve and near the Avacha volcano. The authors listed 22 species, including Jungermannia fusiformis new to Russia. In 1990 I. V. Chernyadjeva collected bryophytes in the South Kamchatian Reserve. The hepatics were identified by A. D. Potemkin and published by Chernyadjeva \& Potemkin (2003), who enumerated 38 species of hepatics. In addition, Potemkin, during his study of other Kamchatian collections, found Kurzia sylvatica (Konstantinova $\&$ al., 1992) a new species for the Russian flora.

The author of this current paper collected liverworts in Kamchatka in 2001 and 2002. Two species from these collections were published as exsiccata in 2003 (Bakalin \& Konstantinova, 2003). The identification of more than 600 specimens from these collections forms the background for this paper.

Prior to the present research only 80 species of liverworts were known from Kamchatka. The current study has increased this to 158 species.

\footnotetext{
1 - Polar-Alpine Botanical Garden-Institute, Kirovsk-6, Murmansk Province, 184256, RUSSIA - Полярно-альпийский ботанический сад-институт, г. Кировск-6, Мурманская область, 184256, Россия - v_bak@aprec.ru
} 


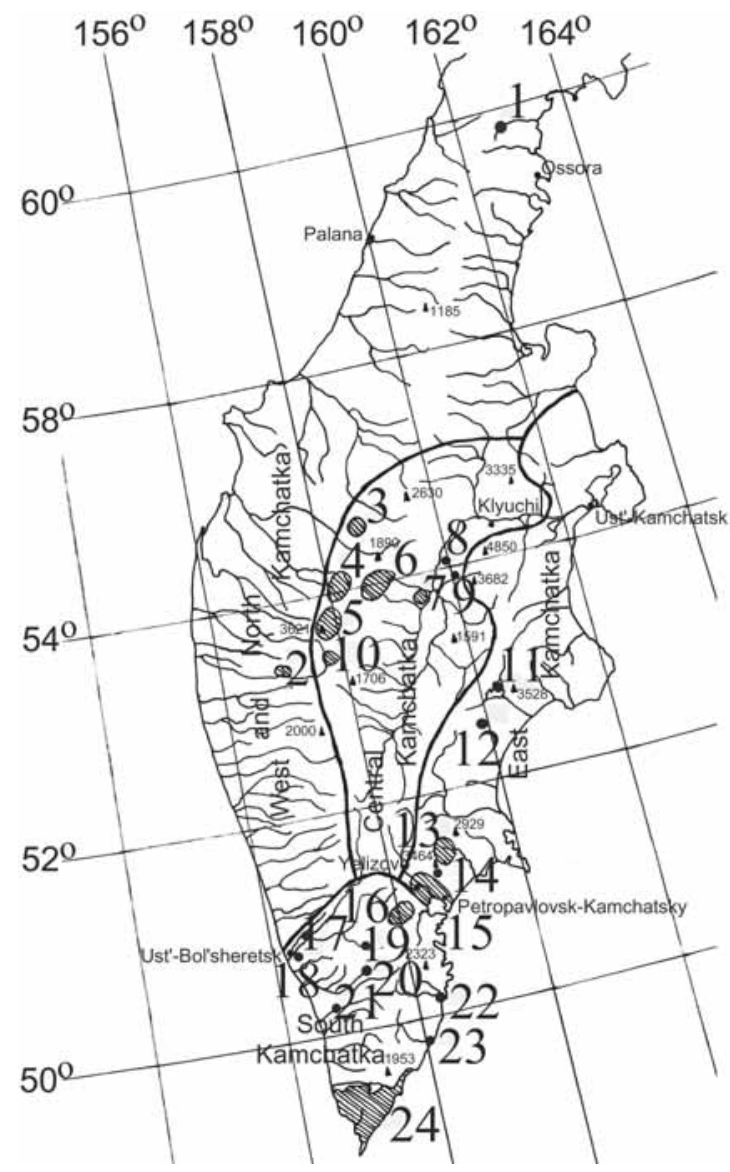

Fig. 1. Collecting localities in Kamchatka:

\section{West and North Kamchatka}

1. Middle course of Kichiga River: Smetanin, 2002.

2. Middle course of the Icha River : Bakalin, coll. 2001.

\section{Central Kamchatka}

3. Oxinsky Hot Springs: Bakalin, coll. 2002.

4. Demshikan Pass: Bakalin, coll. 2002.

5. Okura Pass: Bakalin, coll. 2001, 2002.

6. Neighboring of Esso Settl., Anavgaj Settl, Anauna River, Anauna Mts.: Persson, 1970: Bakalin, coll. 2001, 2002.

7. Lokhmatka (The Middle of Kamchatka River): Bakalin, coll. 2001.

8. Kozyrevsk: Persson, 1970.

9. Tolbachik: Persson, 1970.

10. Shanuch (The Upper Icha River): Bakalin, coll. 2002.

\section{East Kamchatka}

11. Kronotskoye Lake: Persson, 1970.

12. Gejzernaja River and Uzon Caldera: Blagodatskikh, Duda, 2001.

13. Pinochevo and Nalychevo valleys: Blagodatskikh, Duda, 2001; Bakalin, coll. 2001.

14. Avacha vlc.: Blagodatskikh, Duda, 2001.

15. Neigboring of Petropavlovsk-Kamchatsky and Yelizovo Towns (including Mokhovaya, Zavojko): Arnell, 1927; Persson, 1970; Bakalin, coll. 2001.

\section{South Kamchatka}

16. Paratunka Area (including Nachiki Lake): Arnell, 1927; Persson, 1970; Bakalin, coll. 2001.

17. Agashka (Amigacha) River: Persson, 1970.

18. Bol'shaja River and Bol'sheretsk Area: Arnell, 1927; Persson, 1970

19. Bannaja River : Arnell, 1927; Persson, 1970.

20. Opala vlc.: Arnell, 1927; Persson, 1970.

21. Opala River : Persson, 1970.

22. Asacha Bay: Arnell, 1927.

23. Khodutka Area: Arnell, 1927.

24. South Kamchatian Reserve (including Toporkov Isl.): Persson, 1970; Czernyadjeva \& Potemkin, 2003.

The author expects that species could also be

Only a few previously recorded taxa were not re-found and 78 species are new records for the Kamchatka Peninsula. One species is newly recorded for Russia (the first locality outside Japan), two species are new for Asia, 4 are new for East Asia, and two species are recorded for only the second time in Asia. These records are discussed in detail in the checklist.

There is a very complex question: how many species can be added by future studies? As shown in the map (Fig. 1.), collections from Kamchatka are very patchy. Thus, only about 20 species are known from West and North Kamchatka, and of these, two species, (Pleurocladula albescens and Marchantia polymorpha) are only known from locality 1 . In locality 2 hepatic collection was restricted to 1.5-2 hours so the intensity of study does not allow us to say that the liverwort flora is completely known. found in Kamchatka that at present are only known from the Koryak Uplands, whose mountain system extends to the north of the Kamchatka Peninsula. However, it is doubtful if many more temperate species will ever be found in Kamchatka because the severe tundra of the Koryak Uplands impedes migration. Comparison of the floras of the Koryak Uplands (Afonina \& Duda, 1989; Konstantinova \& Kuzmina, 2001) and the Kamchatka Peninsula revealed that the following 18 taxa known from Koryak were not found in Kamchatka: Barbilophozia rubescens (R. M. Schust. et Damsh.) Kartt. et L. Soderstr., Calypogeia neesiana (C. Mass. et Carest.) Müll. Frib., Cephalozia ambigua C. Mass., Cephaloziella arctica Bryhn. et Douin, Eremonotus myriocarpus (Carr.) Lindb. \& Kaal., Lophozia polaris (R. M. Schust.) R. M. Schust., Marsupella commutata 
(Limpr.) H. Bernet, Mesoptychia sahlbergii (Lindb. et Arnell) Evans, Odontoschisma denudatum (Nees) Dumort., Prasanthus suecicus (Gottsche) Lindb., Protolophozia elongata (Steph.) Schljakov, Radula prolifera Arnell, Riccia bifurca Hoffm., R. cavernosa Hoffm., Saccobasis polymorpha (R. M. Schust.) Schljakov, Sauteria alpina (Nees) Nees, Scapania tundrae (Arnell.) H. Buch, S. zemljae S. W. Arnell and Tritomaria scitula (Tayl.) Jörg.

In addition, some Arctic (Marsupella arctica (Berggr.) Bryhn et Kaal., Scapania simmonsii Bryhn et Kaal., etc) and Arctomontane species (Marsupella sprucei (Limpr.) H. Bern., Scapania brevicaulis Tayl., Athalamia hyalina (Sommerf.) Hatt., etc.) could possibly be found on Kamchatka. Some species with amphioceanic and Pacific affinities may be also found (Scapania obscura (Arnell et C. E. O. Jensen) Schiffn., Macrodiplophyllum imbricatum (M.A.Howe) Perss., Solenostoma rubrum (Gottsche ex Underw.) R. M. Schust., etc.). Additional records of some disjunct species are also possible.

In conclusion it is estimated that the number of hepatics known from Kamchatka could increase by 35-40 taxa (about 20\%).

COLLECTING LOCALITIES USED IN CHECK-LIST

Treatment of the floristic districts follows Persson (1970). Comparison of modern geographic names with those used in older literature revealed many discrepancies between old and modern names. Most of these discrepancies arise from differences in transliteration systems (Shadutka is undoubtedly Khodutka, Savoiko - Zavojko, etc.). Some differences result from historic changes of names (Maschuna became Mokhovaya, Agashka - Amigacha, etc.). One site, Akhomten Bay, is probably the modern Asacha Bay. Some sites, such as Sahach vlc. and Berenzojarr, have not been located on recent maps, but both are within South Kamchatka (Persson, 1970). Collecting localities are shown in Fig. 1.

\section{CHECK-LIST}

Nomenclature follows N. A. Konstantinova \& al. (1992), with some recent alterations: Konstantinova \& A. N. Vasiljev (1994), Bakalin (2001), Bakalin \& al. (2001). New taxa for Kamchatka are marked by asterisk. Authors of names are cited as proposed by R. K. Brummit \& Powell C. E. (1992). The synonyms used by R. Grolle \& D. G. Long (2000) are added where concept of genera is different (e. g. Jungermannia s. 1. and Lophozia s. 1.).

Additional information is provided on reproductive structures and collecting localities:

ant. - antheridia

arch. - archegonia

per. - perianthia

spor. - sporogonia

gemm. - gemmae.

W\&NK - West and North Kamchatka

CK - Central Kamchatka

EK - East Kamchatka

SK - South Kamchatka.

*Anastrophyllum sphenoloboides R. M. Schust. - per.

- CK: 6! - One of the most southern localities $\left(56^{\circ} \mathrm{N}\right)$. Previously in East Asia was only found in Chukotka (Afonina \& Duda, 1993).

*Aneura pinguis (L.) Dumort. - EK: 13!; CK: 3!, 4! Anthelia julacea (L.) Dumort. - SK: 22! (Arnell, 1927), 24! (Persson, 1970; Czernyadjeva \& Potemkin, 2003).

A. juratzkana (Limpr.) Trevis. - per., ant., spor. CK: 5!, 6!, 10!; EK: 13!; SK: 16!, 19 (Persson, 1970), 24 (Czernyadjeva \& Potemkin, 2003).

*Apometzgeria pubescens (Schrank) Kuwah. - CK: 5!, 6!; EK: 15 !

Asterella saccata (Wahlenb.) A. Evans. - Without locality (Wahlenberg, 1811).

Barbilophozia barbata (Schmidel ex Schreb.) Loeske - CK: 4!, 5!, 6!, 10!; EK: 15! (Persson, 1970), SK: 24 (Czernyadjeva \& Potemkin, 2003).

B. hatcheri (A. Evans) Loeske - ant., gemm. - CK: 4!, 5!, 6!; EK: 15!; SK: 24 (Czernyadjeva \& Potemkin, 2003).

B. lycopodioides (Wallr.) Loeske - CK: 3!, 5!; EK: 12 (Blagodatskich \& Duda, 2001), 15!; SK: 16!, 20 (Arnell, 1927), EK:

*Bazzania bidentula (Steph.) Steph. - CK: 10!

${ }^{*}$ B. ovifolia (Steph.) Hattori - CK: 10! - The most northern locality in Eurasia.

${ }^{*}$ B. tricrenata (Wahlenb.) Lindb. - CK: 10!

Blasia pusilla L. - gemm. - CK: 3!, 6!; EK: 15! (Arnell, 1927).

Blepharostoma trichophyllum (L.) Dumort. - per., spor., gemm. - CK: 4!, 5!, 6!; EK: 15!; SK: 24 (Czernyadjeva \& Potemkin, 2003); without locality (Wahlanberg, 1811).

Calycularia laxa Lindb. et Arnell - EK: 12 (Blagodatskich \& Duda, 2001), SK: 16!, 24 (Czernyadjeva \& Potemkin, 2003).

Calypogeia azurea Stotler et Crotz - Only literature data: SK: 21 (Persson, 1970; Wahlenberg, 1811), 24 (Czernyadjeva \& Potemkin, 2003).

${ }^{*}$ C. integristipula Steph. - gemm. - CK: 5!,6!

C. muelleriana (Schiffn.) Mull. Frib. - W\&NK: 2!; CK: 4!, 5!, 6!, 10!; EK: 13!; SK: 18 (Persson, 1970), 
24 (Czernyadjeva \& Potemkin, 2003).

${ }^{*}$ C. sphagnicola (Arnell et J. Perss.) Warnst. et Loeske - CK: 5 !

Cephalozia bicuspidata (L.) Dumort. - per., ant., spor., gemm. - CK: 5!, 6!, 10!; EK: 12 (Blagodatskich \& Duda, 2001), 13!, 15!; SK: 18 (Arnell, 1927), 24 (Czernyadjeva \& Potemkin, 2003).

${ }^{*}$ C. connivens (Dicks.) Lindb. - ant., arch. - W\&NK: 2!

C. leucantha Spruce - Only literature data: SK: 18 (Arnell, 1927).

C. lunulifolia (Dumort.) Dumort. - per. - CK: 5!, 6!, 10!; EK: 15!; SK: 18 (Arnell, 1927).

${ }^{*}$ C. pachycaulis R. M. Schust. - per. - CK: 5!; EK!: 15!; SK: 16! - This poorly known species was gathered in a few localities in Kamchatka Peninsula. It grows on soil along paths in birch grass forest, on fine-grained soil in spots of cryogenic origin, in moss-lichens tundra and on rocky banks of streams in brushwood of Pinus pumila Pall. In Eurasia this species is also known from KhamarDaban Range (South Siberia, Konstantinova \& al. 2004). Mixed with Cephalozia bicuspidata, Di plophyllum taxifolium, Marsupella emarginata, Tetralophozia setiformis, Pellia neesiana, Nardia japonica,Plectocolea subelliptica,Blepharostoma trichophyllum.

C. pleniceps (Austin) Lindb. - per. - CK: 5!; EK: 11 (Persson, 1970).

*Cephaloziella arctogena (R. M. Schust.) Konstantinova - ant., arch., per., gemm. - W\&NK: 2; CK: 5!, 6!, 7!; EK: 15!; - The most southern locality in the world (see Konstantinova, 2000).

${ }^{*}$ C. divaricata (Sm.) Schiffn. - ant., arch., per. CK: 5!, 6! ; EK: 15 !

C. rubella (Nees) Warnst. - ant., arch., per., spor., gemm. - CK: 5!, 6!, 7!, 8 (Persson, 1970); EK: 13!, 15!; SK: 24 (Czernyadjeva \& Potemkin, 2003).

${ }^{*}$ C. springera (Lindb.) Warnst. - ant., per., spor. W\&NK: 2!; CK: 6 !

${ }^{*}$ C. uncinata R. M. Schust. - per., ant. - CK: 6!; EK: 15 ! - The most southern locality and the first for East Asia.

Chiloscyphus fragilis (A. Roth) Schiffn. [C. pallescens (Ehrh. ex Hoffm.) Dumort.] - EK: 12 (Blagodatskich \& Duda, 2001).

C. pallescens (Ehrh. ex Hoffm.) Dumort. -EK: 15 (Persson, 1970).

C. polyanthos (L.) Corda - ant., arch. - CK: 6!, 10!; EK: 13!, 15!; SK: 16!, 24 (Czernyadjeva \& Potemkin, 2003).

C. rivularis (Schrad.) Hazsl. [C. polyanthos (L.) Corda]-EK: 15 (Arnell, 1927).

Cladopodiella fluitans (Nees) H. Buch -EK: 15!; SK: 18! (Arnell, 1927).

Conocephalum conicum (L.) Dumort. - CK: 3!, 5!, 10!; EK: 13!, 15! (Arnell, 1927); SK: 16!

C. japonicum (Thunb.) Grolle - EK: 12 (Blagodats- kich \& Duda, 2001); 15!; SK: 22!, 23 (Arnell, 1927).

Crossogyna autumnalis (DC) Scaljakov [Jamesoniella autumnalis (DC) Steph.] - without locality (Persson, 1970)

Diplophyllum albicans (L.) Dumort.- CK: 5!; SK: 24 (Czernyadjeva \& Potemkin, 2003)

*D. obtusatum (R. M. Schust.) R. M. Schust. - per., ant. - CK: 10 - This taxon was described by Schuster (1974) from Minnesota, and occurred only in few localities in North America. This species was recorded for the first time in Eurasia from the Sayan Mts. (South Siberia, Konstantinova \& Vasiljev, 1994). Subsequently it was found in Upper of Bureja River (Konstantinova et al., 2002) and published as exsiccate from Stanovoye Uplands East Siberia (Bakalin \& Konstantinova, 2003). The autoecious inflorescences was described as the main distinguishing feature (Schuster, 1974; Konstantinova \& Vasiljev, 1994). However, in a large collection from East Siberia (used as material for exsiccate) among the majority of autoecious plants some paroecious and dioecious specimens were found, although these plants did not differ from the autoecious ones in any other characters. So, it is possible $D$. obtusatum is only an autoecious race of D. obtusifolium.

D. taxifolium (Wahlenb.) Dumort. - per., ant., gemm. - CK: 4!, 5!, 6!, 10!; EK: 13!, 14 (Blagodatskich \& Duda, 2001), 15!; SK: 16!, 22 (Arnell, 1927), 24 (Czernyadjeva \& Potemkin, 2003).

Fossombronia Raddi sp. - Only literature data: SK: 16 (Arnell, 1927).

*Frullania bolanderi Austin - W\&NK: 2!; CK: 6!, 7!, 10!; EK: 13!, 15!;

${ }^{*} F$. dilatata (L.) Dumort. - CK: 6!

Gymnocolea inflata (Huds.) Dumort. - per., ant. CK: 5!, 10!; EK: 12 (Blagodatskich \& Duda, 2001); SK: 16 (Persson, 1970), 24 (Czernyadjeva \& Potemkin, 2003).

* Gymnomitrion apiculatum (Schiffn.) Müll. Frib. CK: 5 !, 6 !

${ }^{*}$ G. concinnatum (Lightf.) Corda - ant., spor. CK: 5!, 10!

${ }^{*}$ G. corallioides Nees - spor. - CK: 5 !

*Harpanthus flotovianus (Nees) Nees - CK: 5, 6; EK: 13 !

${ }^{*}$ Hygrobiella laxifolia (Hook.) Spruce - per., ant. - CK: 6!, 10!; EK: 13!

*Isopaches bicrenatus (Schmidel ex Hoffm.) H. Buch [Lophozia bicrenata (Schmidel ex Hoffm.) Dumort.] - per., ant., spor., gemm. - CK: 5!, 6!; EK: $13 !, 15$ !

*Jungermannia atrovirens Dumort. - EK: 13!

*J. borealis Damsh. et Váňa - CK: 6 !

*J. eucordifolia Schljakov [Jungermannia exertifolia subsp. cordifolia (Dumort.) Váňa] - ant. - CK: 5!, 10!; EK: 13 ! 
J. exertifolia Steph. -EK: 12 (Blagodatskich \& Duda, 2001); SK: 24 (Czernyadjeva \& Potemkin, 2003).

*J. polaris Lindb. - per., ant. - CK: 6 !

J. pumila With. - per., ant., spor. - CK: 5!, 6!; EK: 12 (Blagodatskich \& Duda, 2001), 13!

Kurzia sylvatica (A. Evans) Grolle - Without locality (Konstantinova et. al., 1992).

*Leiocolea bantriensis (Hook.) Jörg. - CK: 3!

*L. gillmanii (Austin) A. Evans - CK: 6!

L. heterocolpos (Thed. ex Hartm.) H. Buch - gemm. - CK: 5!, 6!, CK, without locality (Persson, 1970).

L. rutheana (Limpr.) Müll. Frib. - CK: 6 (Persson, 1970).

*Lepidozia reptans (L.) Dumort. - CK: 4!

Lophocolea heterophylla (Schrad.) Dumort. W\&NK: 2; CK: 5!, 6!; EK: 13!, 15! (Persson, 1970).

L. minor Nees - gemm. - W\&NK: 2!; CK: 3!, 4!, 5!, 6!; EK: 13!, 15! (Persson, 1970).

*Lophozia excisa (Dicks.) Dumort. - ant., arch., per., spor., gemm. - CK: 4!, 5!, 6!

* L. heteromorpha R. M. Schust. et Damsh. - ant., gemm. - CK: 6! - This extremely poorly known species was gathered in the sources of the Ubojnyj Creek on the bank of an almost dry brook, among mosses. The species is known from northernmost part North America (Schuster, 1969) and in Russia from Chukotka (Bakalin, unpublished). The so named specimens from Yamal Peninsula (Potemkin, 1993) should be referred to $L$. ventricosa sensu lat.

L. lacerata N. Kitagawa - SK: 24 (Czernyadjeva \& Potemkin, 2003).

L. longidens (Lindb.) Macoun - per., ant., gemm. W\&NK: 2; CK: 5!, 6!, 10!; EK: 13!, 15!; SK: 16!, 22 (Persson, 1970).

*L. propagulifera (Gottsche) Steph. - per., ant., spor., gemm.-CK: 4, 5-Study of the type specimen in $\mathrm{JE}$ revealed that it is identical with $L$. latifolia (as it is described by Schuster, 1969) in all essential characters: plants are dioecious (not monoecious, as was written by Schuster, 1969), cells and gemmae size, and shape of androecia, etc. are identical with those of L. latifolia. The synonymy of L. latifolia and L. jurensis was shown by Schljakov (1973). Unfortunately, the type specimens of L . latifolia was unavailable for me (as well as for Schljakov, 1973), so the both L. latifolia and L. jurensis are considered here as provisional synonyms of $L$. propagulifera. The nomenclature is as follows: Lophozia propagulifera (Gottsche) Steph. 1901. Exped. Antarct. Belge, Bot.: 3, 4 (Sept.) Bull. Herb. Boissler (ser. 2) 1:1145 (Oct.) Spec. Hep. 2:128, 139 - Jungermannia propagulifera Gottsche 1890. Neumayer, Int. Polarfrosch., Deutsch. Exped. 2:451 (Type: South Georgia. Bachgrund oberhalb der Pinguinbay. 26 Jan. 1883, coll. Will, det. Gottsche; JE!). - Lophozia jurensis Meylan ex Müll. Frib. 1916. Rabenhorst Kryptogamenfl. Deutschland. (ed. 2) 6(2):767 (Type: Switzerland. “... Schweizer-
Jura: ... Vuarnon bei St. Croix. 1903 coll. et det. Meylan) - L. latifolia R. M. Schust. 1953. Bryologist. 56:258 (Type: USA. "The Point, Grand Marias, Cook County, Minnesota” coll. et det. Schuster).

L. savicziae Schljakov - ant., gemm. - CK: 5!, 6!; EK: 13!; SK: 16!, 24 (Czernyadjeva \& Potemkin, 2003) This species is poorly known outside of Russia and seems to be an occasional component of the alpine coenosis in Kamchatka. It is characterized by numerous partly biconcentric oil-bodies, so its unambiguous identification is only possible when living cells are present for study. Treatment of the species was given by Bakalin (2000).

L. schusteriana Schljakov - ant., gemm. - SK: 16!, 22 (Persson, 1970).

*L. silvicola H. Buch - ant., arch., per., gemm. W\&NK: 2; CK: 4!, 5!, 6!, 10!; EK: 15!; SK: 16!

*L. silvicoloides N. Kitagawa - ant., per., gemm. CK: 5!, 6! - This species is known in East Asia from Japan, Kolyma Uplands and South Primorje; in Europe from Murmansk Region (distribution map was published by Bakalin, 2001).

L. sudetica (Nees ex Huebener) Grolle - per., spor., gemm. - CK: 5!, 6!, 10!; EK: 12 (Blagodatskich \& Duda, 2001), 13!, 14 (Blagodatskich \& Duda, 2001), 15!, SK: 16!, 18 (Arnell, 1927), 24 (Czernyadjeva \& Potemkin, 2003).

L. ventricosa (Dicks.) Dumort. sensu Grolle and Long, 2000, excluding L. silvicola - ant., per., gemm. CK: 4!, 5!, 6!, 10!; EK: 13!, 15!; SK: 16!, 24 (Czernyadjeva \& Potemkin, 2003).

*L. wenzelii (Nees) Steph. - gemm. - CK: 5!, 6!; EK: 13 !

* Macrodiplophyllum microdontum (Mitt.) H.Perss. - CK: 6!

M. plicatum (Lindb.) H. Perss. - gemm. - CK: 4!, 5!, 6!, 10!; SK: 16!, 22 (Persson, 1970).

Mannia pilosa (Horn) Frey et Clark - Without locality (Wahlenberg, 1811).

Marchantia alpestris (Nees) Burgeff [Marchantia polymorpha sensu lat.] - gemm. - CK: 3!, 5!, 6!, 10!; EK: 13!, 15!; SK: 17 (Persson, 1970).

M. polymorpha L. - ant., spor., gemm. - W\&NK: 1 (Smetanin, 2002); CK: 5!, 6!; EK: 12 (Blagodatskich \& Duda, 2001), 13!; SK: 18 (Arnell, 1927), 24 (Czernyadjeva \& Potemkin, 2003).

* Marsupella alpina (Gottsche ex Husn.) H. Bernet CK: 5!, 6! - The most northern locality in East Asia. * M. boeckii (Austin) Kaal. - ant. - CK: 5!; SK: 16! - The most southern locality in East Asia and one of the most southern localities in the world.

M. brevissima (Dumort.) Grolle - CK: 6!; EK: 13!; SK: 22 (Persson, 1970).

* M. emarginata (Ehrh.) Dumort. - ant. - CK: 10!; SK: 16 !

*M. funckii (F. Weber et D. Mohr) Dum. - CK: 5! M. sphacelata (Gieske ex Lindenb.) Dumort. - SK: 
24 (Czernyadjeva \& Potemkin, 2003).

* Metzgeria furcata (L.) Dumort. - per., spor. CK: 10 !

*Moerckia blyttii (Moerch) Brockm. - ant. - SK: 16! - In East Asia this species was only known from Japan (see distribution map in Konstantinova, 2000), so this is the second locality in East Asia.

Mylia anomala (Hook.) Gray - gemm. - W\&NK: 2!; CK: 5!, 6!, 10!; EK: 15!; SK: 18 (Arnell, 1927).

Nardia assamica (Mitt.) Amakawa - EK: 12 (Blagodatskich \& Duda, 2001); SK: 19 (Arnell, 1927, as N. kamtschatica Arnell), 24 (Czernyadjeva \& Potemkin, 2003).

N. breidleri (Limpr.) Lindb. - ant., arch., per., spor. - CK: 5!; EK: 13!, 15!; SK: 24 (Czernyadjeva \& Potemkin, 2003).

N. compressa (Hook.) Gray - SK: 22 (Arnell, 1927).

N. geoscyphus (De Not.) Lindb. - per., ant. - CK: 4!, 5!, 6!, 10!; EK: 13!, 15!; SK: 24 (Czernyadjeva \& Potemkin, 2003).

${ }^{*} N$. insecta Lindb. - ant., arch., per. - CK: 4!, 5!, 6! N. japonica Steph. - ant., arch. - CK: 5!; EK: 12 (Blagodatskich \& Duda, 2001), 13!; SK: 16!; SK: 24 (Czernyadjeva \& Potemkin, 2003).

N. scalaris Gray - SK: 24 (Czernyadjeva \& Potemkin, 2003).

*N. unispiralis Amakawa - CK: 5! - This poorly known species was gathered on the East slope of the Ichinskaya Sopka volcano, in the upper Bol'shoj (Tunnel'nyj) Creek along a stream, with Cephalozia bicuspidata. According to J. Váňa (1976), N. unispiralis is synonymous with and has priority over N. hiroshi Amakawa. In Amakawa's treatment (1959) both species are characterized by dioicous inflorescences and leaves bilobed for $1 / 4-1 / 5$ the length. $N$. unispiralis differed from $N$. hiroshi in obsolete underleaves (versus rather small, but distinct) and in small cells in margin if the leaf 15$18 \mathrm{~mm}$ at apex (versus 22-30). Additionally, $N$. unispiralis has elaters with only one spiral, while sporophyte in $N$. hiroshi have not been found. Váňa (1. c.) did not explain his reasons for merging the two species. However, we do not have access to material to evaluate this synonymy and concur with Váňa's opinion. Sterile plants of this species (or both species) differ from other Nardia in the more or less lophozioid appearance, and are similar to Protolophozia elongata. From the latter N. unispiralis differs in generative structures (mainly in the characteristic perigynium) and fewer oil-bodies (up to 4 in leaf cells versus 15-30 in Protolophozia). This species appears close to $N$. japonica, but differs in small to obsolete underleaves, up to half the stem width, and $1 / 4$ of the leaf length, versus the rather large underleaves of $N$. japonica, almost as wide as the stem and $1 / 2$ to as long as the leaf. This locality is the first one outside Japan. Identification is based on sterile plants.
*Obtusifolium obtusum (Lindb.) S. W. Arnell [Lophozia obtusa (Lindb.) A. Evans] - CK: 5!

Orthocaulis attenuatus (Mart.) A. Evans [Barbilophozia attenuata (Mart.) Loeske]- gemm. - EK: 13!, 15!; SK: 16!, 22 (Persson, 1970).

O. binsteadii (Kaal.) H. Buch [Barbilophozia binsteadii (Kaal.) Loeske] - CK: 5!, 6!; EK: 15!; SK: 18 (Arnell, 1927, as Jungermannia binsteadii).

O. kunzeanus (Huebener) H. Buch [Barbilophozia kunzeana (Huebener) Müll. Frib. - gemm. W\&NK: 2!; CK: 4!, 5!, 6!, 9 (Persson, 1970), 10!

Pellia endiviifolia (Dicks.) Dumort. - ant. - CK: 6!, 10!; EK: 12 (Blagodatskich \& Duda, 2001); SK: 24 (Czernyadjeva \& Potemkin, 2003).

P. epiphylla (L.) Corda - EK: 15 (Arnell,1927); without locality (Wahlenberg, 1811).

P. neesiana (Gottsche) Limpr. - ant. - CK: 3!, 4!, 5!, 6!; EK: 13! (Blagodatskich \& Duda, 2001), 15 (Arnell, 1927); SK: 16!, 21(Arnell, 1927), 24 (Czernyadjeva \& Potemkin, 2003).

*Peltolepis quadrata (Saut.) Müll. Frib. - spor. EK: 13 !

*Plagiochila porelloides (Torrey ex Nees) Lindenb. - CK: 10!; EK: 15!

Plectocolea hyalina (Lyell) Mitt. [Jungermannia hyalina Lyell]- ant. - CK: 6!; EK: 15!; SK: 19 (Arnell, 1927).

P. infusca Mitt. (Jungermannia infusca (Mitt.) Steph. -SK: 24 (Czernyadjeva \& Potemkin, 2003).

$P$. obovata (Nees) Lindb. [Jungermannia obovata Nees]- ant., arch. - CK: 10!; SK: 24 (Czernyadjeva \& Potemkin, 2003).

${ }^{*}$ P. subelliptica (Lindb. ex Kaal.) A. Evans [Jungermannia subelliptica (Lindb.ex Kaal.) Levier] - ant., arch., per. - CK: 5!, 6!, 10!; EK: 13!; SK: 16 !- Since Schljakov (1981) this species was included in $P$. obovata in the Russian literature and consequently its Russian distribution is poorly known.

P. vulcanicola Schiffn. [Jungermannia vulcanicola (Schiffn.) Steph.] - per. - CK: 5!; EK: 12 (Blagodatskikh, Duda, 2001); SK: 24 (Czernyadjeva \& Potemkin, 2003).

Pleurocladula albescens (Hook.) Grolle - per. W\&NK: 1 (Smetanin, 2002); CK: 4!, 5!, 6!; EK: 12 (Blagodatskich \& Duda, 2001), 13!, 14 (Blagodatskich \& Duda, 2001), 15!; SK: 16!, 22 (Arnell, 1927), 24 (Czernyadjeva \& Potemkin, 2003).

*Preissia quadrata (Scop.) Nees - spor. - CK: 3!, 4!, 5!, 6 !

*Protolophozia debiliformis (R. M. Schust.) Konstantinova [Lophozia debiliformis R. M. Schust.] - gemm. - CK: 13! - This is a new species for East Asia and the most southern locality in the world. The record of species for Kamchatka in Konstantinova (2000) is a mistake in compilation of the map (Konstantinova, pers. comm.).

Ptilidium californicum (Aust.) Pears. - per. - SK: 16!, 20 (Persson, 1970), 24 (Czernyadjeva \& 
Potemkin, 2003).

P. ciliare (L.) Hampe - CK: 4!, 5!, 6!, 7!, 18 (Arnell, 1927), 24 (Czernyadjeva \& Potemkin, 2003).

P. pulcherrimum (Weber) Vain. - per. - CK: 5!; EK: 12 (Blagodatskich \& Duda, 2001), 13!, 15!

* Radula complanata (L.) Dumort. - gemm. - EK: 15! * Riccardia latifrons (Lindb.) Lindb. - CK: 4, 5, 6

* Riccia lamellosa Raddi - CK: 6! - This mainly Mediterranean species is found in South Europe (Greece, Italy, France, Spain and Portugal) and in North Africa (Morocco, Algeria, Tunisia, Tripoli, Ethiopia). It is also known from Middle Asia, China and the New World: Argentina, USA and Mexico (Schuster,1992; Ladyzhenskaya,1962; Pii ppo,1999). This is the most northern locality for this species.

Ricciocarpos natans (L.) Corda - EK: 15 (Arnell, 1927).

* Saccobasis polita (Nees) H. Buch [Tritomaria polita (Nees) Jorg.] - ant. - EK: 13!

* Scapania curta (Mart.) Dumort. - ant., gemm. CK: 6!; EK: 13!

S. cuspiduligera (Nees) Muell. Frib. - gemm. - CK: 6!; EK: 13!; SK: 22 (Persson, 1970).

S. brevicaulis Taylor - EK: 15 (Persson, 1970).

*S. glaucocephala (Taylor) Austin - gemm. - CK:

5 !, 7! - This rare species is a newly recorded in East Asia.

*S. hyperborea Jorg. - gemm. - CK: 5!

S. irrigua (Nees) Nees - per., ant., gemm. - CK: 5!, 6!; EK: 13!; SK: 24 (Persson, 1970).

${ }^{*} S$. lingulata H. Buch - gemm. - CK: 6!

*S. mucronata H. Buch - gemm. - CK: 5!, 6!; EK: 15!

${ }^{*}$ S. obcordata (Berggr.) S. W. Arnell - EK: 13! This is the most southern record in the Northern Hemisphere.

S. paludicola Loeske et Müll. Frib. - gemm. - CK: 5!; EK: 13!, 15!, SK: Berenzojarr (Persson, 1970).

S. paludosa (Müll. Frib.) Müll. Frib. - ant. - CK: 4!, 5!, 10!; EK: 13!, EK: 15 (Persson, 1970).

${ }^{*}$ S. parvifolia Warnst. [S. scandica] - per., ant., gemm. - CK: 4!, 5!, 6!

* S. scandica (Arnell et H. Buch) Macvicar - per., ant. - EK: 15!

S. subalpina (Nees ex Lindenb.) Dumort. - ant., per., gemm. - CK: 5!, 6!, 10!; EK: 12 (Blagodatskich \& Duda, 2001), 13!; SK: 22 (Arnell, 1927).

S. uliginosa (Lindenb.) Dumort. - CK: 5!; EK: 12 (Blagodatskich \& Duda, 2001); SK: 24 (Czernyadjeva \& Potemkin, 2003).

*S. umbrosa (Schrad.) Dumort. - per., spor. - CK: 5! S. undulata (L.) Dumort. - gemm. - CK: 4!, 5!, 6!, 10!; EK: 13!; SK: 22 (Arnell, 1927).

* Schistochilopsis grandiretis (Lindb. ex Kaal.) Konstantinova [Lophozia grandiretis (Lindb. ex Kaal.) Schiffn. - gemm. - CK: 6!

${ }^{*} S$. hyperarctica (R. M. Schust.) Konstantinova [Lophozia hyperarctica R. M. Schust.] - gemm. - CK: 4!, 6! This poorly studied arctic species is known from Archangelsk Province (northern Europen Russia),
Arctic Alaska, Canada and North Greenland (see distribution map in Konstantinova, 2000). Recently it was recorded also from Komi Republic (North-East Europe, Russia, see Dulin, et al., 2003). This find is the most southern in the world and a new record for Asia.

S. incisa (Schrad.) Konstantinova [Lophozia incisa (Schrad.) Dumort.] - ant., per., spor., gemm. CK: 4!, 5!, 10!; EK: 13!, 15!; SK: 16!, 22 (Arnell, 1927), 24 (Czernyadjeva \& Potemkin, 2003).

S. opacifolia (Culm. ex Meyl.) Konstantinova [Lophozia opacifolia Culm. ex Meyl.] - per., gemm. - CK: 5!; EK: 13!; SK: 16!, 24 (Czernyadjeva \& Potemkin, 2003).

* Solenostoma caespiticium (Lindenb.) Steph. [Jungermannia caespiticium Lindenb.]- per., spor., gemm. - CK: 5!, 10! - It is the southernmost localities in Asia.

S. confertissimum (Nees) Schljakov [Jungermannia confertissima Nees] - CK: 5!; EK: 12 (Blagodatskich \& Duda, 2001).

S. fusiformis (Steph.) R. M. Schust. (J. fusiformis Steph.) - EK: 12 (Blagodatskich \& Duda, 2001).

*S. gracillimum (Sm.) R. M. Schust. [Jungermannia gracillima $\mathrm{Sm}$.] - arch. - EK: 15! - Species is recorded for the first time in Asia.

*S. jenseniana (Grolle) Bakalin comb. nov. Jungermannia jenseniana Grolle, Oesterr. Bot. Z. 111: 190. 1964.] - per. - CK: 5!

* S. sphaerocarpum (Hook.) Steph. [Jungermannia sphaerocarpa Hook.] - EK: 13!

* Sphenolobus minutus (Schreb.) Berggr. [Anastrophyllum minutum (Schreb.) R. M. Schust.] - ant., arch., per., gemm. - W\&NK: 2!; CK: 4!, 5!, 6!, 10!; EK: 15!; SK: 16!; without locality (Wahlenberg, 1811).

*S. saxicola (Schrad.) Steph. [Anastrophyllum saxicola (Schrad.) R. M. Schust.] - CK: 6!

Targionia hypophylla L. - spor. - CK: 3!; without locality (Wahlenberg, 1811) - This is the northernmost locality in East Asia.

*Tetralophozia setiformis (Ehrh.) Schljakov - CK: 5!, 6!; SK: 16 !

*Tritomaria exsecta (Schmidel) Loeske - gemm. CK: 10 !

*T. exsectiformis (Breidl.) Loeske - per., gemm. CK: 6!

*T. quinquedentata (Huds.) H. Buch - ant. W\&NK: 2!; CK: 4!, 5!, 6!, 10 !

\section{ACKNOWLEDGEMENTS}

I am very grateful to Mrs. E. Kungu (UK) for correcting of English in the manuscri pt.I thank Prof. N. A. Konstantinova for help with identification of some Marsupella and Scapania species, and the curator of JE for loan of Lophozia propagulifera type specimens. The work was partly supported by Russian Foundation for Basic Researches, grants 00-04-48874 and 03-04-49304. I also thank Mrs. A. V. Dul'chenko - a fearless lady and my colleague on the most difficult routes by horses in Kamchatka expedition. 
[AFONINA, O. M., J. DUDA] АФОНИНА, О. М., Й. ДУ ДА 1989. К флоре печеночников Северной Корякии. [On the liverworts flora of North Koryakiya] Hовости cuст. низи. pacm. [Novosti Sist. Nizsh. Rast] 26: 147-149.

[AFONINA, O. M., J. DUDA] АФОНИНА, О. М., Й. ДУ ДА 1993. Печеночники Чукотки. - [Liverworts of Chukotka] Бот. журн. [Bot. Zhurn.] 78(3): 77-93.

AMAKAWA, T. 1959. Family Jungermanniaceae of Japan. I. - J. Hattori Bot. Lab. 21: 248-291.

ARNELL, N. W. 1927. Lebermoose aus Kamtschatka. Hedwigia 67: 110-112.

BAKALIN, V. A. 2000. Notes on Lophozia I. What is Lophozia silvicola Buch var. grandiretis Buch et S. Arnell (Hepaticae: Lophoziaceae). - Arctoa 9: 111-114.

BAKALIN, V. A. 2001. Notes on Lophozia III. Some taxonomic problems in Lophozia sect. Lophozia. - Arctoa 10: 207-218.

[BAKALIN, V. A., N. I. MOLOKOVA, T. N. OTNYUKOVA] БАКАЛИН, В. А., Н. И. МОЛОКОВА, Т. Н. ОТНЮКОВА 2001. К флоре печеночников Тоджинской котловины (Республика Тува, Южная Сибирь). - [On the liverworts flora of Todzha Valley (Tuva Republic, South Siberia)] Arctoa 10: 19-26.

BAKALIN, V. A., N. A. KONSTANTINOVA 2003. Hepaticae Rossica Exsiccata. Fasc. I. (N 1-25) - Kirovsk, Polygraf, 17.

[BLAGODATSKIKH, L. S., J. DUDA] БЛАГОДАТСКИХ, Л. С., Й. ДУ ДА 2001. О флоре печеночников полуострова Камчатка. - [On hepatic flora of Kamchatka Peninsula] Hовости сист. низи. pacm. [Novosti Sist. Nizsh. Rast.] 34: 218-220.

BRUMMIT, R. K. \& C. E. POWEL 1992. Authors of plant names. - London, Royal Botanic Gardens, Kew.

[CZERNYADJEVA, I. V. \& A. D. POTEMKIN] ЧЕРНЯДЬЕВА, И. В., А. Д. ПОТЕМКИН 2003. Мохообразные Южно-Камчатского природного заказника - [The Bryophytes of South-Kamchatian Nature Reserve]. В кн.: Флора и растительность Южной Камчатки [In: Flora i Rastitel'nost' Yuzhnoj Kamchatki] Петропавловск-Камчатский, Камчатский печатный двор [PetropavlovskKamchatsky, Kamchatsky Pechatny Dvor]: 73-98.

[DULIN, M. V., KONSTANTINOVA, N. A., BAKALIN, V. А.] ДУЛИН, М. В., КОНСТАНТИНОВА, Н. А., БАКАЛИН В. А. 2003. К флоре печеночников республики Коми. - [То the liverwort flora of the Komi Republic] Бот. журн. [Bot. Zhurn.] 88(3): 45-52.

GROLLE, R., D. G. LONG. 2000. An annotated check-list of the Hepaticae and Anthocerotae of Europe and Macaronesia. - J. Bryol. 22: 103-140.

[KONSTANTINOVA, N. A.] КОНСТАНТИНОВА, Н. А. 2000. Анализ ареалов печеночников Севера Голарктики. - [Distribution patterns of the North Holarctic hepatics] Arctoa 9: 29-94.

KONSTANTINOVA, N. A., POTEMKIN, A. D., SCHLJAKOV, R. N. 1992. Check-list of the Hepaticae and Anthocerotae of the former USSR. - Arctoa 1: 87-127.

KONSTANTINOVA, N. A. \& A. N. VASILJEV 1994. On the hepatic flora of Sayan Mountains (South Siberia). Arctoa 3: 123-132.

[KONSTANTINOVA, N. A. \& E. Y. KUZMINA] KOHCTAHТИНОВА, Н. А., Е. Ю. КУЗЬМИНА 2001. К флоре печеночников Корякии (Северо-восток России). - [On the hepatic flora of Koryakiya (North East of Russia)] Arctoa 10: 103-114.

[KONSTANTINOVA, N. A., V. A. BAKALIN \& A. D. POTEMКIN] КОНСТАНТИНОВА Н. А., В. А. БАКАЛИН, А. Д. ПОТЕМКИН 2004. Cephalozia pachycaulis R. M. Schust. (Hepaticae, Cephaloziaceae) - новый для России вид печеночника из Сибири и с Камчатки. [Cephalozia pachycaulis R. M. Schust. (Hepaticae, Cephaloziaceae) - a new for Russia liverwort from Siberia and Kamchatka] Бот. журн. [Bot. Zhurn.] 89 [in press].

KONSTANTINOVA, N. A., V. A. BAKALIN, A. D. POTEMKIN \& M. S. IGNATOV 2002. Hepatic flora of the Upper Bureya River. - Arctoa 11: 393-398.

[LADYZHENSKAYA, К. І.] ЛАДЫЖЕНСКАЯ, К. И. 1962. Riccia lamellosa Raddi и R. papillosa Moris - новые виды для флоры CCCP - [Riccia lamellosa Raddi and R. papillosa Moris - new species for the USSR flora] Труды отд. спор. раст. БИН АН СССР [Trudy Otd. Spor. Rast. Bot. Inst. Akad. nauk SSSR] 14: 252-262.

PERSSON, H. 1970. Contribution to the bryoflora of Kamchatka. - Revue Bryologique et Lichenologique 37(2):209-221.

PIIPPO, S. 1990. Annotated catalogue of Chinese Hepaticae and Anthocerotae. - J. Hattori Bot. Lab. 68: 1-192.

POTEMKIN, A. D. 1993. The Hepaticae of the Yamal Peninsula, west Siberian Arctic. - Arctoa 2: 57-101.

[SCHLJAKOV, R.N.] ШЛЯКОB, P.H. 1973. Систематические заметки по семейству Lophoziaceae Cavers. [Systematic notes on the Lophoziaceae Cavers family] Hoвoсти сист. низи. pacm. [Novosti Sist. Nizsh. Rast] 10: 287-302.

[SCHLJAKOV, R. N.] ШЛЯКОВ, Р. Н. 1980. Печеночные мхи Севера СССР Том. 3. [Hepatics of the North of USSR. Vol. 3.] - Л., Наука [Leningrad, Nauka], 188.

[SCHLJAKOV, R. N.] ШЛЯКОВ, Р. Н. 1981. Печеночные мхи Севера СССР Том. 4. [Hepatics of the North of USSR. Vol. 4.] - Л., Наука [Leningrad, Nauka], 212.

SCHUSTER, R. M. 1969. The Hepaticae and Anthocerotae of North America East of the Hundredth Meridian. Vol. 2. New-York - London, Columbia University Press, XII+1062.

SCHUSTER, R. M. 1974. The Hepaticae and Anthocerotae of North America East of the Hundredth Meridian. Vol. 3. - New York-London, Columbia University Press, 880.

SCHUSTER, R. M. 1992. The Hepaticae and Anthocerotae of North America East of the Hundredth Meridian. Vol. 6. - Chicago, Field Museum of Natural History, 937.

SCHUSTER, R.M. \& K. DAMSHOLT 1974. The Hepaticae of West Greenland. - Meedelelser om Grönland 199(1): 1-373 + 80 maps

[SMETANIN, A. N.] СМЕТАНИН, А. Н. 2002. Экосистемы лососевой реки Кичиги (Северо-восточная Камчатка) [Ecosystems of the salmon's river Kichiga (NorthEast Kamchatka) ] - Петропавловск-Камчатский, КамyamГТИ [Petropavlovsk-Kamchatsky, KamchatGTI], 188.

VÁŇA, J. 1976. Studien über die Jungermannioideae (Hepaticae) 10. Nardia. - Folia Geobot. Phytotax. 11: 367-425.

WAHLENBERG, G. 1811. Kamtschadalische Laub- und Lebermoose, gesammelt auf der russischen Entreckungstreise von dem Herrn Hofrath Tilesius. - Magazin für die neuesten Endeckungen in der gesammten Naturkunde 5: 289-297. 